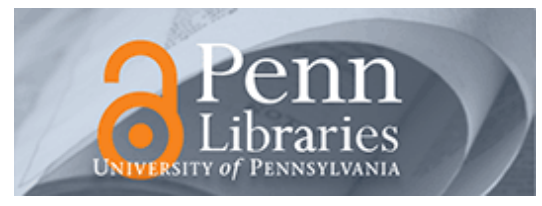

University of Pennsylvania

ScholarlyCommons

September 2003

\title{
Mixed-Signal Calibration of Pipelined Analog-Digital Converters
}

\author{
Sameer R. Sonkusale \\ Texas A\&M University \\ Jan Van der Spiegel \\ University of Pennsylvania, jan@seas.upenn.edu
}

Follow this and additional works at: https://repository.upenn.edu/ese_papers

\section{Recommended Citation}

Sameer R. Sonkusale and Jan Van der Spiegel, "Mixed-Signal Calibration of Pipelined Analog-Digital Converters", . September 2003.

Copyright 2003 IEEE. Reprinted from Proceedings of the 2003 IEEE International SOC [Systems-on-Chip] Conference, pages 327-330.

Publisher URL:http://ieeexplore.ieee.org/xpl/tocresult.jsp?isNumber=27830\&page=5

This material is posted here with permission of the IEEE. Such permission of the IEEE does not in any way imply IEEE endorsement of any of the University of Pennsylvania's products or services. Internal or personal use of this material is permitted. However, permission to reprint/republish this material for advertising or promotional purposes or for creating new collective works for resale or redistribution must be obtained from the IEEE by writing to pubs-permissions@ieee.org. By choosing to view this document, you agree to all provisions of the copyright

This paper is posted at ScholarlyCommons. https://repository.upenn.edu/ese_papers/46

For more information, please contact repository@pobox.upenn.edu. 


\title{
Mixed-Signal Calibration of Pipelined Analog-Digital Converters
}

\author{
Abstract \\ Least-Mean-Squares (LMS) based mixed-signal scheme for self-calibration of pipelined Analog-Digital \\ Converter $(A D C)$ is proposed. The technique uses an elegant continuous reference update algorithm to \\ correct for gain errors and offset errors in a pipeline stage with minimal area and power overhead. \\ Simulation results show the effeciency of the scheme for resolution of greater than 13bits in a CMOS \\ process.

\section{Comments} \\ Copyright 2003 IEEE. Reprinted from Proceedings of the 2003 IEEE International SOC [Systems-on-Chip] \\ Conference, pages 327-330. \\ Publisher URL:http://ieeexplore.iee.. rg/xpl/tocresult.jsp?isNumber=27830\&page=5 \\ This material is posted here with permission of the IEEE. Such permission of the IEEE does not in any way \\ imply IEEE endorsement of any of the University of Pennsylvania's products or services. Internal or \\ personal use of this material is permitted. However, permission to reprint/republish this material for \\ advertising or promotional purposes or for creating new collective works for resale or redistribution must \\ be obtained from the IEEE by writing to pubs-permissions@ieee.org. By choosing to view this document, \\ you agree to all provisions of the copyright
}




\section{Mixed-Signal Calibration of Pipelined Analog-Digital Converters}

\author{
Sameer Sonkusale \\ Dept. of Electrical Engineering \\ Texas A\&M University \\ Email: sameer@ee.tamu.edu
}

\author{
Jan Van der Spiegel \\ Dept. of Electrical and Systems Engineering \\ University of Pennsylvania \\ Email: jan@seas.upenn.edu
}

\begin{abstract}
Least-Mean-Squares(LMS) based mixed-signal scheme for self-calibration of pipelined Analog-Digital Converter (ADC) is proposed. The technique uses an elegant continuous reference update algorithm to correct for gain errors and offset errors in a pipeline stage with minimal area and power overhead. Simulation results show the effeciency of the scheme for resolution of greater than 13bits in a CMOS process.
\end{abstract}

\section{INTRODUCTION}

Pipelined Analog-to-Digital Converters(ADC) architectures are very popular in the design of high-resolution and highspeed ADCs used in broadband communication systems. One such application is a design of a direct conversion receiver, where a high performance ADC enables multi-mode functionality capable of supporting various communication standards, thereby lowering system cost. However, ADCs designed in digital CMOS technology for reasons of low-cost and higher integration do not offer high resolution because of poor capacitor matching, low amplifier gains attributed to lowering supply voltages, charge injection errors, offset errors and poor noise performance. In such cases, self-calibration is always used to push the resolution performance of the ADC [1],[2],[3],[4]. All these calibration schemes mostly correct for errors only due to poor capacitor matching. However they fail to account for the finite gain error of the operational amplifier which has become common-place in today's CMOS technologies with lowering supply voltages. Recently a few digital calibration schemes based on Least-Mean-Squares(LMS) technique have been proposed to correct for errors introduced by finite amplifier gain and capacitor ratio mismtach in a pipeline stage [5],[7]. However these schemes require complex digital processing hardware and do not render itself to easy system on chip implementations. Some of these schemes even need a o separate calibration signal added to the input which sacrifices the converter's useful dynamic range [7]. This paper describes an improved mixed-signal calibration scheme for a pipelined ADC using an LMS based adaptive reference-update algorithm which does not have aforementioned drawbacks.

\section{ONE-Bit PER STAGe Pipeline A/D Converter}

A simplified block diagram of a conventional 1-bit per stage, pipelined $\mathrm{ADC}$ is shown in the figure 1 . It consists of a sample-and-hold amplifier, a coarse ADC, a coarse DAC and a gain stage. The most significant bits are resolved by

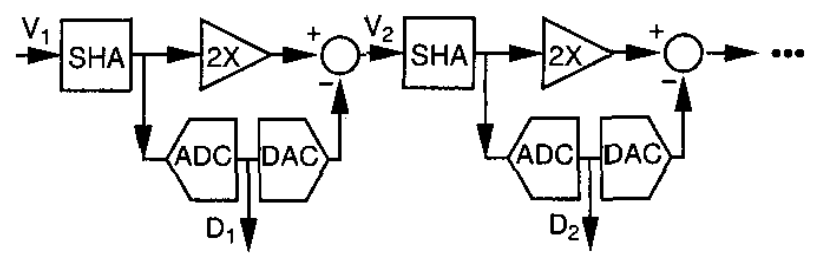

Fig. 1. N-stage 1-bit per stage pipelined ADC prototype

the stages earlier in the pipeline and the least significant bits are resolved later in the pipeline. The most favorable reason for the use of the pipelined ADC for high speed applications is that it's complexity scales linearly with the resolution of the converter (compared to Flash type converters). Since most significant bits are resolved in earlier stages, the overall resolution of the ADC is mostly limited by the first few stages in the pipeline. To understand the sources of error in a pipeline stage, consider a most conventional switched capacitor implementation as shown in figure 2. A single ended circuit is shown for simplicity. $V_{\text {refp }}$ is the positive reference voltage and $V_{\text {refn }}$ is a negative reference voltage $\left(V_{\text {refp }}=-V_{\text {refn }}=V_{\text {ref }}\right)$. The resolvable input range of the converter is given by $2 V_{\text {ref }}$. Each stage consists of

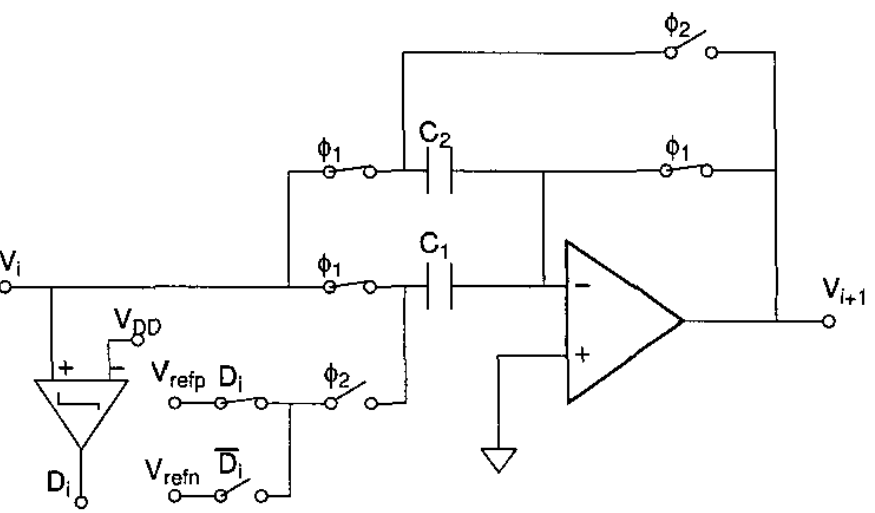

Fig. 2. Switched capacitor 1-bit pipeline stage

two nominally equal capacitors $C_{1}$ and $C_{2}$, an operational amplifier(op-amp), and a comparator. During the sampling phase $\phi_{1}$, the comparator produces a digital output $D_{i}$ : 


$$
D_{i}= \begin{cases}1 & \text { if } V(i) \geq V_{t h} \\ -1 & \text { if } V(i)<V_{t h}\end{cases}
$$

where, $V_{t h}(=0)$ is the threshold voltage defined midway between $V_{r e f p}$ and $V_{r e f n}$. the bipolar representation of $D_{i}$ is used for notational convenience. During the multiply-by-2 and subtract phase, the above circuit generates a residue voltage output $V_{i+1}$ given by:

$$
\begin{aligned}
V_{i+1} & =K\left[\left(1+\frac{C_{1}}{C_{2}}\right) V_{i}+\frac{C_{1}}{C_{2}} D_{i} V_{\text {ref }}\right] \\
K & =\frac{A_{0}}{1+\frac{C_{1}}{C_{2}}+A_{0}}
\end{aligned}
$$

where, the parameter $K$ is an op-amp gain error coeffecient (ideally unity) and $A_{0}$ is the finite op-amp gain. This output residue voltage is then passed to the next stage $i+1$, and the same operation continues. As evident from the above expression, the accuracy of the overall converter depends on the accuracy of the residue output generated by pipeline stages (especially early in the pipeline). Ideally, we expect the residue output voltage to be:

$$
V_{i+1}=2 V_{i}-D_{i} V_{r e f}
$$

From expression 3 and 4 , we see that gain errors are introuduced in the overall transfer chracteristic because of the term $K$ attributed to finite amplifier gain and the term $\frac{C_{1}}{C_{2}}$ which deviates from the ideal value of 1 due to poor capacitor matching. This usually limits the overall resolution of the ADC to 8-10 bits without calibration in today's CMOS process. Charge injection errors have been neglected, since they can be easily corrected through the use of bottom-plate sampling technique and differential operation.

\section{BASIC IDEA OF THE PROPOSED SCHEME}

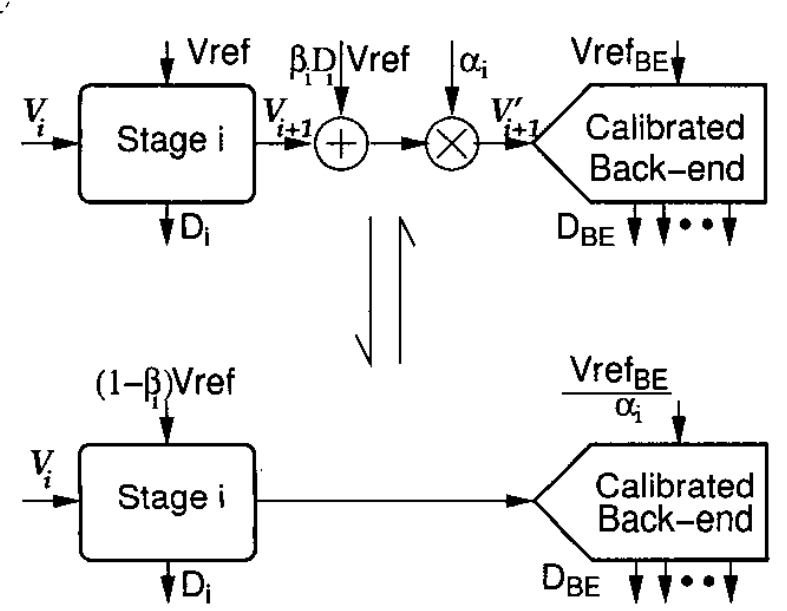

Fig. 3. Basic principle of mixed signal calibration through equivalence of analog processing and the proposed reference manipulation scheme

To correct for gain errors introduced in the pipeline, the residue output of each non-ideal stage in the pipeline can be processed in an analog domain using a set of parameters $\left(\alpha_{i}, \beta_{i}\right)$ for each stage $i$ that essentially perform linear translation of the residue output.

$$
V_{i+1} \quad \rightarrow \quad V_{i+1}^{\prime}=\alpha_{i}\left(V_{i+1}+\beta_{i} D_{i} V_{\text {ref }}\right)
$$

This principle is illustrated in the figure 3 . The stage $i$ is the $i^{\text {th }}$ stage of the pipeline which is under correction and the $B a c k$ - end refers to the following stages in the pipeline. If the value of $\alpha_{i}, \beta_{i}$ for stage $i$ can be set as following, then the modified residue output $V_{i+1}$ will resemble the ideal residue output.

$$
\alpha_{i}=\frac{2}{K\left(1+\frac{C_{1}}{C_{2}}\right)}, \quad \beta_{i}=\frac{K}{2}\left(\frac{C_{1}}{C_{2}}-1\right)
$$

A digital scheme using a slow but high resolution $\mathrm{ADC}$ (SHADC) to estimate these parameters alongwith digital post processing was shown by the same authors [5] [6]. In that implementation, a slow high-resolution $\mathrm{ADC}$ was used to perform parameter estimation of the correction parameters $\alpha_{i}, \beta_{i}$. One of the critical problems with such an implementation was the dependence of the accuracy of calibration on the performance of the SHADC. Also, the technique needed high speed multipliers to perform error compensation in digital domain.

A direct analog scheme as shown in top section of the figure 3 will be difficult to implement due to speed and power constraints. The basic idea of this paper is to perform error compensation in analog domain through manipulation of reference voltages to each pipeline stage. The process of multiplying a residue output by a gain term $\alpha_{i}$ can be shown to be equivalent to scaling the reference voltages of all the following pipeline stages by $1 / \alpha_{i}$ [4]. Similarly the process of adding an offset term $\beta_{i} D_{i} V_{\text {ref }}$ to the residue output can be shown to be equivalent to adding a term $-\beta_{i} V_{\text {ref }}$ to the the reference voltage of the stage under correction. This equivalence is illustrated in the figure 3 .

The parameter estimation process for $\alpha_{i}, \beta_{i}$ could be performed using the scheme proposed in [5] which requires a use of an SHADC. However, to reduce power consumption, and taking notice of the fact that in most applications, an $\mathrm{ADC}$ is not being used constantly, the use of an additional complex converter with higher accuracy is not justified. Hence a mixed signal scheme with an elegant novel reference update algorithm has been proposed which performs parameter estimation during start-up and/or when the $\mathrm{ADC}$ is idle.

\section{Complete Mixed-Signal IMPlementation of The Calibration SChEme}

Figure 4 shows the reference update scheme for the stage $i$, that needs calibration. The technique uses a lowresolution, monotonic DAC (CDAC), that behaves like a digital potentiometer around a nominal reference value, to provide reference input to the pipeline stages. During the inactive or idle mode of the $\mathrm{ADC}$, the input to the stage $i$ is forced to either fullscale $V_{\text {ref }},-V_{\text {ref }}$ or ground. For 


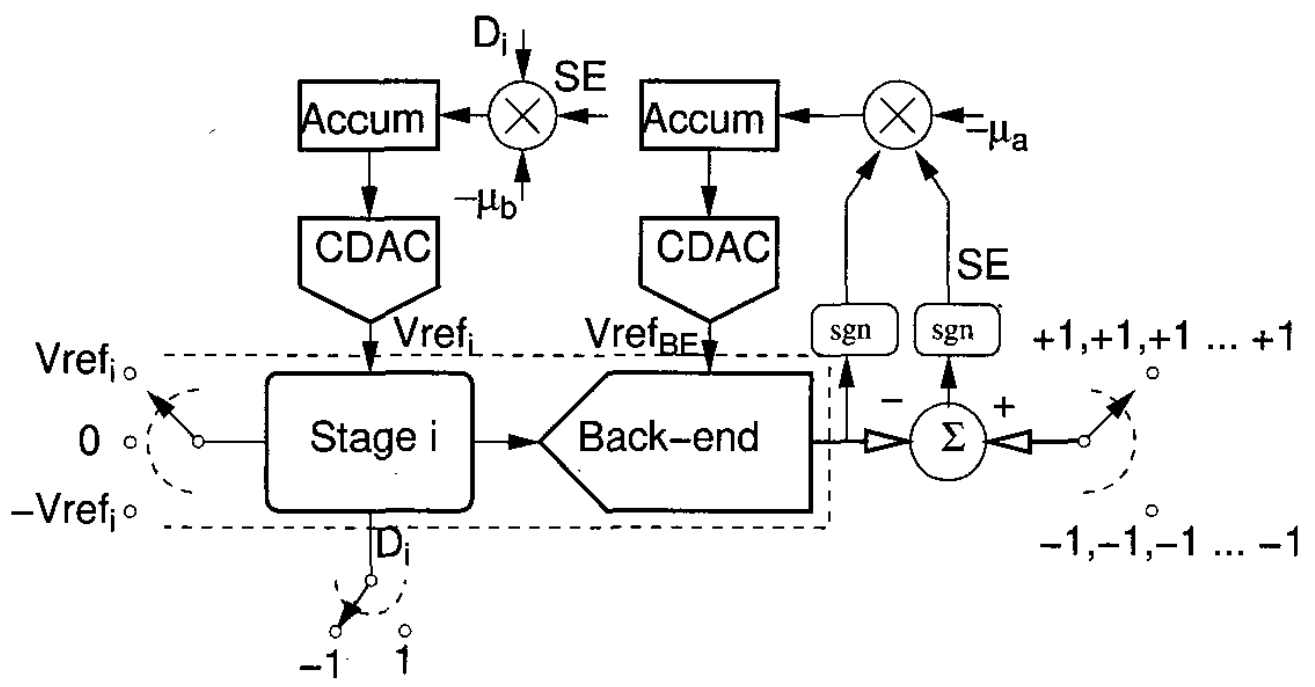

Fig. 4. Mixed Signal Implementation of the Calibration Scheme

the grounded input, the digital output of the stage is also forced to either $D_{i}=1$ or $D_{i}=-1$. This generates a total of four different known input values to the stage $i$. For each of these four input values, the only possible set of outputs from the following pipeline stages (shown as a block $B a c k$ - end in the figure 4) in an ideal situation is either all 1 's $+1,+1,+1 \ldots+1$ or all -1 's $-1,-1,-1 \ldots-1$. Any deviation from the ideal values of the back-end suggests gain and offset error for the pipeline stages $i$ onwards.

A sign-sign LMS algorithm is used to continuously updates the reference voltage $V r e f_{B E}$ for the back-end and the reference voltage $V$ ref $f_{i}$ of the stage $i$ under calibration by randomly or sequentially switching the inputs to the suggested four input configurations. Let the sign of the deviation of the digital output of the back-end from it's ideal value be given by the $S E$, as shown in the figure 4. The update algorithm for $V r e f_{B E}$ and $V r e f_{i}$ at time index $n$ is given below:

$$
\begin{array}{ll}
\operatorname{Vref}_{B E}[n]= & \operatorname{Vref} f_{B E}[n-1]-\mu_{a} S E \operatorname{sgn}\left(D_{B E}\right) \\
\operatorname{Vref}_{i}[n]= & \operatorname{Vref} f_{i}[n-1]-\mu_{b} S E D_{i}
\end{array}
$$

$\mu_{a}$ and $\mu_{b}$ are the update sizes for the above sign-sign update algorithm. Their value is chosen as small as possible, considering the signal and quantization noise power constraints, the excess mean squared error bounds and the convergence accuracy. The step size can be made programmable for faster convergence [6]. For reasons mentioned in section II, only the first few stages of the pipeline will need calibration. Once the parameters for the stage $i$ are estimated, similar estimation can be carried out for stage $i-1$ and so on. A recursive estimation process beginning with the least significant Bit(LSB) stage that needs calibration until the Most Significant Bit (MSB) stage is ideal for estimation purposes since the Back - end would resemble an ideal converter for the given accuracy. The error term in
Eq. 6 now corresponds to only the error contribution from stage $i$. To increase the accuracy of the scheme, however, the quantization noise power in $D_{B E}$ needs to be reduced. This can be achieved by using a few extra pipeline stages towards the end of the ADC.

The CDAC in the figure 4 should be monotonic over the possible input range. The linearity of CDAC is not critical, since the update loop automatically accounts for the nonlinearity of this DAC. The resolution of the DAC may be small of the order of $6-8$ bits with monotonicity equal to the overall resolution of the converter. This low-resolution, mononic potentiometer DAC can be easily implemented using current steering thermometer-decoded arrays [8], which guarantees inherent monotonicity and small step sizes. The power consumption of such a DAC is indeed very small and would only be a small percentage of the overall power consumption pie. Further power savings can be achieved by sharing the CDAC between adjacent stages of the pipeline. The multiplication operation in the figure 4 is merely an addition or subtraction operation by a value of $\mu_{a}$ (or $\mu_{b}$ ) depending on the polarity of the other inputs $\left(S E, D_{i}\right.$ and $D_{B E}$ ). Hence the overall calibration loop only needs an accumulator, adder and a few gates for its implementation. Compared to the digital post-processing implementation scheme proposed in [5], this scheme does not require any post processing of the raw output, since the digital output of the back-end is already a corrected version. Thus the need for high speed multipliers and adders have been eliminated. This also significantly reduces the digital switching noise coupled to the analog section of the chip.

In the above discussion, we have neglected the input dependence of the non-idealities like the finite op-amp gain. High linearity from amplifiers can be easily obtained by trading the absolute value of the gain for linearity in the design. As indicated from the algorithm, the absolute value of 
the gain of the amplifiers is a non-issue within limits imposed by the common mode rejection and other constraints. Some of the other not-so serious non-idealities like comparator offsets and op-amp offsets can be minimized using bottom-plate sampling, differential operation and comparator redundancy in the pipeline stage [3][9].

\section{Simulation Results}

A 13-bit resolution ADC (intended to run at $100 \mathrm{MHz}$ ) is simulated at a behavioral level. There are 16 stages in the pipeline. The technology is assumed to have a capacitor ratio mismatch of $1-3 \%$ and the op-amp gain in the range of 800 1200. Pipelined ADC desgined without calibration in such a technology would yield only 8 bit resolution even at low speed. In the simulation setup, the CDAC of the figure 4 is assumed to have a resolution of 8 bits with step-size of 14 bit resolution. Since the technology yields raw resolution of 8 bit, only first 5 stages of the pipeline will need calibration to generate an output of 13 bit resolution. As suggested in the proposed scheme, the $\mathrm{ADC}$ is calibrated during an inactive or idle mode by switching the input of the ADC randomly to assume one of the four input configurations (see section IV) and running the update algorithm before convergence is reached. The figure 5 shows that the Integral Non-Linearity (INL) of the pipelined ADC has improved from a value of $\pm 64 \mathrm{LSB}$ to $\pm 0.5 \mathrm{LSB}$. The Differential Non-Linearity (DNL) plot of the figure6(a) indicates that DNL has improved by around 252LSBs.

Figure 6(b) shows the normalized value of the reference voltages for the sixteen stages of the pipeline before and after running the the reference update algorithm. The dashed line indicates the normalized value before calibration.

\section{CONCLUSION}

A mixed signal scheme consisting of a digital referenceupdate algorithm and an analog error-compensation scheme through manipulation of reference voltage is suggested. The scheme does so with mininmal analog and digital overhead making it suitable for embedded applications. The simulation results for a 13 bit converter has been shown. The results indicate significant improvement in the integral and differential linearity performance of the ADC.

\section{REFERENCES}

[1] Un-Ku Moon and B. S. Song, Background Digital Calibration Techniques for Pipelined ADC's, IEEE Trans Circuits Syst. II, vol. 44, no. 2, pp. 102109, February 1997.

[2] J. Ingino and B. Wooley, A continuously calibrated 12-b, 10-MS/s, 3.3$V A / D$ Converter, IEEE J. of Solid State Circuits, vol. 33, no. 12, pp. 1920-1931, December 1998.

[3] Hae-Seung Lee, A $12-b 600 \mathrm{Ks} / \mathrm{s}$ Digitally Self-Calibrated Pipelined Algorithmic $A D C$, IEEE J. of Solid State Circuits, vol. 29, no. 4, pp. 509-515, April 1994.

[4] K. Nagaraj, Area-effecient Self-Calibration Technique for Pipe-lined Algorithmic $A / D$ Converters, IEEE Trans Circuits Syst. II, vol. 43, pp. 540-544, July 1996

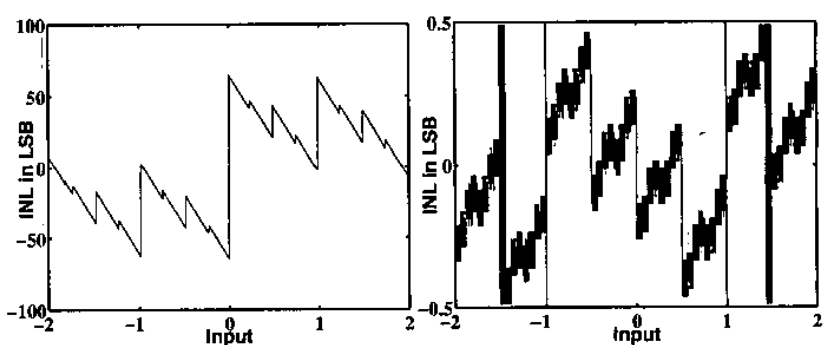

Fig. 5. INL error profile: capacitor ratio mismatch $1-3 \%$, op-amp gain 800 1200 a) Before and b) After Calibration

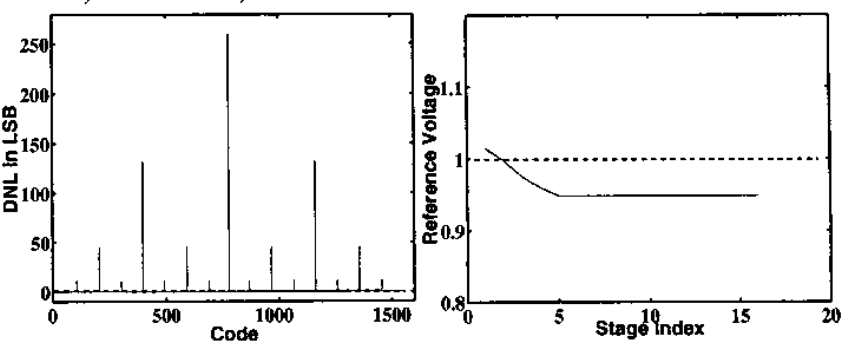

Fig. 6. a) DNL error profile before Calibration (the darker line at 0 shows DNL after calibration) b) The Reference values before (dotted line) and after (dark line) calibration

[5] Sameer R Sonkusale, Jan Van der Spiegel and K. Nagaraj, Background Digital Error Correction Technique for Pipelined Analog-Digital Converters, Proc. International Symp. on Circuits and Syst. 2001, vol. 1, pp. $408-411$.

[6] Simon Haykin, Adaptive Filter theory, Prentice Hal], 1986.

[7] Jun Ming and Stephen H. Lewis,An 8-bit 80-Msample/s Pipelined Analogto-Digital Converter With Backgroun Calibration, IEE J. of Solid State Circuits, vol. 36, no. 10, pp. 1489-1497, October 2001.

[8] B. Razavi, Principles of Data Conversion, Piscataway, IEEE Press, 1994.

[9] S. H. Lewis et al., A 10-b 20-Msample/s Analog-to-Digital Converter. IEEE J. Solid State Circuits, vol, 27, no. 3, pp. 351-357, March 1992. 